\title{
A therapeutic dilemma: symbolic versus literal approach whilst working with adolescent patients, shown with the use of clinical examples.
}

\author{
Maria Barbara Lokaj*, Maria Teresa Aydemir \\ Outpatient Clinic for Families, Private Practice, Poland
}

*Correspondence to: Maria Barbara Lokaj, Jungian Analyst, Clinical Psychologist, Outpatient Clinic for Families, Private Practice, Przedwiosnie 9/20, Katowice, Silesia 40-319, Poland; Tel: 48 693634783; E-mail: m.lokaj@interia.pl

Citation: Lokaj MB, Aydemir MT. A therapeutic dilemma: symbolic versus literal approach whilst working with adolescent patients, shown with the use of clinical examples. J Psychol Cognition. 2017;2(4):237-243.

Received date: November 17, 2017; Accepted date: December 12, 2017; Published date: December 19, 2017

Copyright: (C) 2017 Lokaj MB, et al. This is an open-access article distributed under the terms of the Creative Commons Attribution License, which permits unrestricted use, distribution, and reproduction in any medium, provided the original author and source are credited.

\begin{abstract}
The purpose of this paper is to discuss a dilemma often faced by psychotherapists from various therapeutic schools: a choice between a symbolic and literal approach. Authors, Jungian Analysts and Clinical Psychologists with long-term clinical experience, focused in their article on that how this dilemma manifests in analytical psychotherapy of adolescent patients. In order to illustrate this issue, Authors introduced two clinical examples from their analytical work with adolescents. The article has been prepared based on the material presented by the Authors during the 23rd International Conference on Adolescent Medicine \& Child Psychology "Unveiling Novel Therapies and Developmental Strategies to Help Children Grow Better", organised by Conference Series on the 28th-29th of September 2017, in Berlin. Some of the patients' data has been changed due to confidentiality.
\end{abstract}

Keywords Adolescent patients, Dilemma, Psychotherapy. Introduction

A number of our adolescent patients have presented significant emotional deficiencies resulting from the lack of enough parental care and support. In these situations, therapists often become a 'substitute parent'. Our dilemma, as therapists, is how to keep a symbolic and analytical attitude facing these significant deficiencies and the needs of our young patients. This dilemma is critical, as the period of adolescence is an especially important and difficult moment in one's life - a time of a transition from childhood to early adulthood. This time of a transition requires guidance from an adult figure helping them to enter into early adulthood. The question is how far can or should the therapist fulfil this role?

It is a dilemma for an every therapist and counsellor who works with children and adolescents because of the needs presented by their young patients. On the one hand, the need to experience being guided by an adult during the time of the transition from childhood to adulthood. But on the other hand, in the case of adolescent patients with emotional deficits, these needs also stem from the earlier stages of development and concern the experience of parental care, which was not sufficiently provided in their childhood. As these needs are usually expressed indirectly, a therapist is therefore under especially strong pressure from their teenage patient's need. Thus, from a therapeutic point of view, it is always a complexed situation which creates a serious dilemma: whether and how to respond to the patient's needs. But for us, Jungian Analysts, due to the nature of the analytical work, this dilemma is acutely profound.

\section{Methods of Work in Analytical Therapy}

The particular nature of analytical work consists of methods used in analytical therapy, which result from the analytical theory. As Jungian Analysts, we use the method consisting on the work with the unconscious psyche of our patients using metaphors and basing on the paradigm of symbolisation. As both of us work according to the development approach in the Post-Jungian psychology, called 'the London School', our methods of work consist also of providing patients a special therapeutic setting, taking care of the boundaries in the relationship and using transference and countertransference.

\section{Unconsciousness as a paradigm of 'depth psychology'}

Analytical theory is a part of the bigger stream called 'depth psychology'. This approach assumes an existence of the unconsciousness. According to this approach, unconsciousness manifests itself in a person's dreams, erroneously carried-out actions, verbal mistakes, fantasies, neurotic symptoms, etc. There is an assumption in this approach that unconsciousness strongly influences a person's conscious life. 
Citation: Lokaj MB, Aydemir MT. A therapeutic dilemma: symbolic versus literal approach whilst working with adolescent patients, shown with the use of clinical examples. J Psychol Cognition 2017;2(4):237-243.

\section{The specifics of the Jungian approach: symbolisation}

The core of Jungian approach is about looking for meaning: the meaning of patient's symptoms, of patients' difficulties, of synchronistic events in analysis and life, and above all - the meaning of symbols which appear in patient's dreams. The situation of lowering the threshold of consciousness, called 'participation mystique', enables a patient to achieve a deeper contact with an unconscious part of their psyche, the area of 'alive' symbols. A therapist's task is to allow their patient to contact with this area, as symbols have a reviving influence on the conscious psyche, helping the patient to modify their attitude to life and thus enabling them to adapt to their current life situation.

\section{The analytical setting and boundaries}

There are many elements that compose of what is called 'the psychotherapy setting': a combination of elements in the psychotherapy framework that are set up to provide continuity, consistency and containment, in order to help the patient feel safety and 'held' emotionally by their therapist. Beside a constant place of meetings - the consulting room - there are other elements of the analytical setting including the consistent session time and duration, the consistent frequency of sessions, the way of paying the fees, the reaction of the therapist in the case of absences, breaks and cancellations, the ways of communication, confidentiality and numerous other parameters which we deliver to our patients.

The analytical boundaries offered by us, can often be difficult for our adolescent patients to tolerate. It is because this kind of work confronts our patients with the feeling of frustration. Their frustration in analytical therapy comes from three main sources:

A. Boundaries connected with limits of the therapeutic frame work (e.g. the length of the therapy sessions - a session finishes at the same time despite the fact that it started later than usually because a patient was late)

B. The limits in how much work the therapist can do for the patient (e.g. the therapist can talk with the patient about their problems but doesn't solve their problem for them)

C. The therapist role is to comment on the patient's stories and images by giving interpretations and clarifications instead of giving reassurance which is often craved by the patient.

\section{Transference and counter-transference}

In the analytical approach, transference is understood as all projections of a patient onto the therapist. These projections mostly consist of emotions and imaginations related previously with patient's parental figures. Counter-transference is understood in this approach as all therapist's feelings and emotional reactions for patient's contents, behaviour, feelings and dreams. Our skills of working with the transference and counter-transference arrive from our professional training which included also our own individual long-term analysis. Because of the permanent contact with the contents of unconscious psyche, the very important part of our methods is to regularly consult with our analytical supervisors during the entire time of our professional career (not only during the time of being a trainee, as it is usually in the case of other psychotherapeutic schools). Our method of work consists of devoting our attention to understand the information which derives from the transference and counter-transference feelings. Then, basing on this understanding, we deliver the analytical interpretations to our patients.

\section{The Main Sources of the Therapeutic Dilemma in Analytical Work}

The main axis of our therapeutic dilemma in analytical work running between two attitudes which an Analyst can (consciously or unconsciously) identify with:

\section{'Cruel Witch - The Child Eater' - A symbolic role}

In the most of old cultures there was a figure of a shaman who guided teenagers during the time of their initiation into an adulthood by enabling them to contact with their culture symbols related with this life threshold. A teenager's parents could not act like the guide as they were considered being 'too soft' for their children to pay this role. In these terms, the character of the cruel witch from the Brothers Grimm's' fairy tales "Hanzel and Gretel" is understood as a figure of a shaman running an initiation of teenagers. The shaman-witch 'eats' children because a 'child part' in a teenager's psyche has to 'die' to enable them to become adult. Human psyche did not changed during ages and a figure of the shaman-guide is still necessary for young people. An Analyst, because of their knowledge about symbols, can play this role successfully.

\section{'Friendly Foster Parent' - A literal role}

Teenagers with a history of severe deficits of parental care expect to receive a portion of a missing parental care from other adults, including their therapist. Thus, while meeting with the adolescent patients, strong transferential feelings and expectations during the course of therapeutic process, an Analyst, in their counter-transferral feelings, might be often tempted to play the role of a 'good foster parent' who tries to compensate the emotional deficits caused by real parents. It is very difficult to not be tempted by being seen as a 'good' figure while facing with the adolescent patients' complains and anger during the painful time of crossing their life threshold.

Using the analytical methods of the work with the unconscious psyche activates patients' contact with symbols and unconscious images. As adolescents have just left their childhood, the time of fairy tales and unconscious images, they now need to put their feet on solid ground in the real world. Thus, paradoxically, the analytical work based on symbolisation can help adolescent patients in this process of grounding.

\section{Clinical Examples}

The title dilemma has been demonstrated through two clinical examples: 


\section{Clinical example one: 'Natalie' - "I prefer to die than to be average"}

An adolescent girl, whom I called 'Natalie', was in an individual psychotherapy with me for over 3 years. According to the Polish psychological literature, the time of adolescence is not limited only to the teenage years but it starts earlier and finishes later. The time of adolescence is divided as following:

- Pre-adolescence, puberty: for girls 10-11, for boys 11-12

- Early adolescence: for girls 13-16, for boys 13-17

- Late adolescence: for girls 17-20, for boys 18-22

When applied to the therapy, 'Natalie' was a 17 year old pupil of a private college, having problems with completing the term. Thus, according to the division set out above, she was in her late adolescence. 'Natalie' suffered from low mood, strong anxieties causing the situation that she was even afraid to get up from her bed and problems with relationships with her peers: she was very lonely having no friends. She also failed high schools and changed schools many times.

'Natalie' was the only child of her parents. She grew up in a well-off family, with high standard of life. Her father, a businessman, was very successful at work but usually absent, being involved very much in his business. Her mother did not work. 'Natalie' always wears expensive clothes and had very high expectations regarding her style of life. Her parents bought her a car. 'Natalie' felt a strong anger toward her parents but she was also seeking emotional reassurance from them.

When 'Natalie' was at out, her mother phoned her several times a day. 'Natalie's' mother was always over-caring and allowed her daughter for almost everything. The Patient described the situation at her parents' home so: her father was lying on the sofa, 'Natalie' was sitting nearby and her mother was running around them and catering to their every need. 'Natalie' grew up with the impression that her father was very lazy and helpless, so she felt contempt for her father. She also felt contempt for her mother because her over-caring attitude and was on the opinion that her mother was stupid.

'Natalie' spent her childhood in the house full of bowls of sweets standing on tables in every room. Thus, 'Natalie' used to eat sweets all the time and became addicted on them. Her parents also ate sweets every day and ignored their daughter's addiction.

As a child, 'Natalie' was told many times by her father that only educated people could have a chance to become rich and only rich people could have any meaning and be respected in the society. When 'Natalie' was 12 years old, her father used to give her physics books for 15 years old. 'Natalie' could not fulfil her father's orders to complete the exercises from the book. Then her father sneered at her. 'Natalie' felt stupid and helpless and hated her father. When 'Natalie', as a child, asked her father a question, her father used to give her a very complicated answer which she was not able to understand and felt hopeless again.

The first year of therapy, was a time of struggling with boundaries. 'Natalie' was fighting with her teachers in the college by not delivering her projects on time and with me by being late to her sessions. She perceived a clock on the wall in my consulting room as her oppressor and me as an oppressive male figure who expected from her things which were impossible to do. I contained 'Natalie's' anger and her attacks on boundaries. My role was to be patient but assertive at the same time. It was like being with a 3 years old toddler who was testing her limits.

In the 2nd year, 'Natalie' started to cooperate with me and changed her attitude to her school: she changed her college that time from a private for a public high school (in Poland, the level of public colleges and universities is usually much higher than the level of private high schools, so it costed her more effort to learn in a public school than in a private one). First time in her life, 'Natalie' completed the year without repeating any exam. She also increased her social circle, starting seeing regularly three new peer-friends.

At the time when 'Natalie' was doing practically nothing, lying in her bed with her head covered with a blanket, she developed grandiose fantasies that she would soon become a millionaire who would open her own fashion house and become famous. In the third year of her therapy, when 'Natalie' has already started to verify her real capabilities, she became very disappointed and frustrated. She started to develop a negative transference, blaming me for taking her addictive fantasies and emotional 'drugs' away from her. 'Natalie' could not accept the fact that she was not the best student in her class and however hard she worked; she was unable to fulfil her own expectations. She started developing strong feelings of hatred and contempt towards herself and to have suicidal thoughts. She felt a huge rage to the world and herself that she was not such an outstanding person as she expects herself to be. In the attacks of rage, 'Natalie' shouted and kicked doors in her flat and throws objects. She also tried to kill a dog with her speeding car and felt satisfaction and triumph when the animal was escaping in panic.

'Natalie' often repeated: "I prefer to die than to be average." and: "If I had to work in a supermarket, I would kill myself." To show me that she was serious about that, 'Natalie' described the situation when she was sitting on the outside windowsill of her apartment on the eighth floor.

When 'Natalie' has changed her life situation in a positive way and overcame her anxieties, she started feeling worse. This is an example of a negative therapeutic reaction. Realised that she is not so great as she saw herself in her fantasies, she started to punish me for this. She became sarcastic and arrogant towards me, rejecting and denigrating all my interpretations. She repeated that everything what I said was useless for her. She wanted to leave her therapy but felt she was in the trap. Once, she said: "You took my leg and now I can't go away". As the 'leg', she understood her faith in her grandiose fantasies which were a sense of her life since her childhood.

In the transference, the Patient acted out and reversed the situation from her childhood: 'Natalie' played the role of her humiliating father and I was playing the role of humiliated child - 'Natalie'. The Patient demanded concrete answers for 
Citation: Lokaj MB, Aydemir MT. A therapeutic dilemma: symbolic versus literal approach whilst working with adolescent patients, shown with the use of clinical examples. J Psychol Cognition 2017;2(4):237-243.

her questions and simple 'prescriptions' how to solve her problems, refusing to even start to struggle with my interpretations. She repeated with an ironic voice: "I know all this but how does this help me?"

The problem in this situation as a therapist, was to find a middle ground where I was not doing everything for 'Natalie' like her over-caring mother but at the same time was not like her father, giving his daughter tasks too difficult for her to achieve and making her feel hopeless.

In the counter-transference, firstly, I found myself trying to fulfil 'Natalie's' expectations, running around her with a plate of soup, like her mother did. I tried to simplified my interpretations, as 'Natalie' complained that she could not understand anything and that my interpretations were "too abstractive" for her. I was also considering to give her some kinds of 'advice' and even gave her comments how she could subtly modify her behaviour regarding her social relationships, as she used to complain all the time that she did not know how to keep long-term relationships with her peers. But, despite all my efforts, the Patient was still disappointed and denigrated my attempts to help her. So, I realised that I was doing all this only to avoid feeling helpless as a therapist.

Then, I started to offer my Patient more challenges during our sessions. I gave her comments and interpretations which required her effort to digest them. But I realised, that I started to feel impatient and developed strong irritation toward 'Natalie' - in the counter-transference, I became somehow her cruel father. So, I started to push myself to develop empathy for 'Natalie'. In order to help both of us - 'Natalie' and me - to get out of the trap of the vicious circle of humiliation and retaliatory triumph, I suggested her to limit the time of her therapy. I have suggested the following framework: we will work together during next 3 months, and then we will decide whether we are able to continue our work. If not - I will find 'Natalie' another therapist.

The Patient agreed for this idea and was grateful to me because she stopped feeling imprisoned by me. She hated me but in the same time she was afraid to stay without any therapy and didn't know where to find help. I also started to offer 'Natalie' some kinds of explanation of her intrapsychic situation, based on simple images. 'Natalie' found this way of working helpful for her. But after some weeks, she returned to complain for my 'useless' role and demand receiving advice and concrete solutions from me. When we were considering realising our previous idea of terminating the therapy, 'Natalie' suddenly, 'in a wonderful way', regained her 'lost' ability to understand my interpretations and became a cooperating patient, with an unexpectedly high level of an ability to reflect on her problems.

Thinking about 'Natalie's' therapeutic process, I am still having doubts how much she really was not able to symbolise and understand my interpretations and how much she just manipulated refusing to chew the interpretations by herself, imitating her 'lazy' father lying on the sofa and expecting me to feed her with a spoon...

\section{Clinical example two: 'Alister' - “Only I'm keeping myself warm"}

My patient is a 15 year old boy whom I will refer to as Alister. He started analysis with me 3 years ago. His history is a story of early and hard abandonment.

He is an adopted child. When he was only a few months old he was left by her biological parents. At the age of 9 months he was adopted by her current parents.

Alister's parents divorced when he was 8 years old. Both parents had new relationships, but not stable and long-term ones. Now, his father has a child from one of his more recent relationship. In the past, his father abused drugs and alcohol. The father's addiction to drugs and alcohol were the main reason for the divorce. Since the divorce, the father has finished rehabilitation for addictions. Alister's mother has been under psychiatric care for many years having taken tablets because of depression. Her mood is very changeable and is easily put into a bad mood.

Mother is very critical with Alister. Some coldness between them is visible and they have difficult story together including physical violence (they fought with each other). The difficulties in their relationship were a reason to send Alister to psychological and psychiatric treatment when he was 12 .

Alister has decided that he wants to leave home when he is 18 because of the cold relationship with his mother. He said: "I wonder if she realises that I will not have any contact with her when I will be an adult?"

Father seems more responsive for Alister needs. When I asked him for help to find a new school for Alister - father responded actively. He seems more engaging and caring. Alister has learning difficulty and failed secondary school. He started a new school. Mother was very disappointed because of his failure and called him stupid.

Alister brought to his analysis problems with obsessive thoughts about possible abandonment by his girlfriend. These thoughts are very exhausting for him. Alister often can't sleep and he is very anxious at night. $\mathrm{He}$ is also anxious about relationships with his peers and imagines rejection from them. This anxiety is very visible in how he behaves in his new school.

Alister presented himself to me as someone surprisingly mature and without illusions. It has been very difficult and bitter for him realising that he will not get longed get affection and support from his mother. His attitude towards his parents is a mixture of anger and disappointment but on the other hand his respect and care is visible. My impression was that despite his whole difficult history, he was sometimes more mature than his parents, more caring and understanding for them than they are for him.

Alister said once: "Only I'm keeping myself warm". It was in context of absence of his father in his everyday life and the moodiness and anger of his mother. This short, moving sentence shows briefly the core of his relationship with his actual parents but maybe it also echoes his early abandonment 
by his biological parents. For me, this statement was incredibly sad, insightfully showing me his loneliness.

This quote from Winnicot [2] seems to fit very well for Alister:

"While growing process in adolescence is in progress, responsibility must be taken by parental figures. If parentfigures abdicate, then adolescents must make a jump to a false maturity and lose their greatest asset: freedom to have ideas and act on impulse" (Winnicot, 1966, p.176)

During his analysis, Alister said a few times "I can only rely on you and I trust you'". He really cared about our relationship, in a way that other adolescents do not care. I believe and hope that I gave him good enough container but I tried not to exclude his parents. My impression was that his parents were busy with their lives and simply gave him under my 'psychological care'.

Alister needs more psychological 'holding' than just one session per week but has no money for additional sessions. He initiated to send me text messages and I allowed him to do this. He used this privilege just times of crisis- the exchange of messages between us were just in order to calm him down. These text conversations were only short exchanges as he did not abuse this form of contact but he has a feeling that I am somewhere and accessible. From analytic point of view, it is questionable because of boundaries and limits set.

Alister brought something very symbolic to his analysis. $\mathrm{He}$ got a tattoo on his back, it was a tattoo of a wing. The tattoo was not really clear and the Patient referred to it sometime as a wing of a bird and sometimes as a wing of an Angel. The wings is a very vivid symbol for his survival and strength, reminding him that he will get through difficult moments in his life, reminding him about his own strength.

I believe that that my role is to help Alister to grow, step by step, a symbolic attitude. In the face of a lack of good enough adult support, I wanted to provide him something what is missing, something which helps him to go through this difficult period of him. I hope this is not too much and not too little. I invited his parents to cooperate with me but I believe that help in becoming an adult is still needed for him in face of parental deficiency.

\section{Discussion}

Usually in the transference, the Analyst is a kind of parent for the patient. It is particularly true whilst working with adolescent patients. When the parents are not supportive or sometimes even destructive, then adolescents' longing for a good parent becomes very strong. There is an unconscious pressure on the Analyst to do more, to go further, and to be as a foster parent. "Whether we like it or not, Analysts are likely to be unconsciously affected by their patients [1]."

The influence exerted by the patients on their therapists is visible in the therapists' counter-transferential feelings. In the analytical method of work, these feelings are used as an invaluable source of information: the therapist's inner reflection on their own counter-transferential feelings helps them to understand better the patient's unconscious emotional dynamics. As Paula Heimann said: “...the analyst's emotional response to his patient within the analytic situation represents one of the most important tools for his work. The analyst CT (Counter-Transference) is an instrument of research into the patient's unconscious".

This emotional pressure is much stronger in the analysis of adolescents than in the analysis of adults. This is what was experienced by the Analysts in their therapeutic relationships with 'Alister' and 'Natalie'.

What is important for an adolescent is to grow and develop a symbolic attitude. The importance of the symbolic attitude during the time of adolescence relates with the developmental threshold, as described in the previous part of this article. During this special time of transition, an adolescent person prepares to begin to participate in the adult space of culture. Thus, as Rosemary Gordon said, symbols “....could come to act for us as a bridge connecting our understanding of the inner world of fantasy with the outer world of culture [2]".

The symbolic attitude can be enriched through playing, imagination and creating new ideas. When an adolescent has to control the family dynamics and focus on their parents' problems, then there is no space for them to freely play and imagine. Alister seems to be too adult, too literal, even his relationship with his girlfriend seems to be too serious; it's rather more like a marriage than an adolescent relationship. Winnicot said: "Child may suddenly need to become responsible because (for instance) of the break-up of a family (...) such a child must be prematurely old and must lose spontaneity and play and carefree creative impulse. This is false maturity" [3].

To take a symbolic attitude, it is necessary to be able to operate with a symbolic function [2]. The symbolic function is understood here as: an 'adult' ability to operate in the symbolic space, called, in the terms of the Jungian approach, the 'transcendent function' [4] and also as a basic ability to symbolise [2]. This basic ability arises during the process of symbol formation which starts at the time of early childhood. This inner process which takes place in a child psyche has been described comprehensively in the works of Winnicott [5]. There are three main stages of this process: the first stage when only a literal, physical presence of a maternal figure is able to deliver a reassurance to a child, the second stage - when a child reassures themselves by using a 'transitional object' (by cuddling their teddy-bears or blankets which represent a maternal figure) and the third stage - when a child is able to reassure themselves by imagining an inner image of the maternal figure which already exist in the child psyche [5].

The Analyst's dilemma during her work with Alister, focused around the issue how much the Analyst should be literally present in Alister's life and how much should she be available for him. This is why she had doubts regarding the text messages exchanging between her and her patient. A certain 'portion' of frustration connected with a physical absence of a mother is necessary to enable a child to pass the stages the process of symbol formation. The issue of the proper 'doses' of reassurance and frustration is connected with the idea of a 
Citation: Lokaj MB, Aydemir MT. A therapeutic dilemma: symbolic versus literal approach whilst working with adolescent patients, shown with the use of clinical examples. J Psychol Cognition 2017;2(4):237-243.

'good enough mother' [5]. But when a mother is not available for a child when the child is too young to tolerate her absence, then an image of a terrifying maternal figure replaces an image of a 'good mother' in the child psyche [6]. It is the case of Alister who was abandoned as an infant. Despite her previous doubts, Analyst agreed to exchanged text messages with Alister, to offer her patient a chance to re-built his inner image of a 'terrifying mother'. The text messages can be understood in this context as an intermediate stage between a reassurance delivered literally to Alister by his Analyst playing a role of his 'foster parent' and a reassurance 'delivered' to the patient by his 'transitional object' (the text of the message stayed with the patient after his Analyst sent it him).

Therefore, the story of Alister's tattoo can be seen as a continuation of this process of symbol formation. Tattooed wing could be understood as a creative impulse. This image reminded Alister the strength of his survival, his identity, his individuality. It was so when Alister associated his tattoo with a bird's wing. At the other time he associated it with a wing of an angel. A figure of a good, protecting angel symbolises a figure of a good parent. Because of his painful story, it is difficult for Alister to have inside of him image of something good so he wanted this image very close: on his skin. Thus, we can understand the patient's tattoo as a specific form of a 'transitional object'.

And what about Natalie who bullies her Therapist by the blackmail that she will kill herself or somebody else if she doesn't become a genius millionaire? Grandiose self and narcissistic vulnerability is a central feature of adolescence. Adolescents usually have an ideal which helps them to transit into adulthood. But Natalie has been fixed on her omnipotent sense of self and refuses to see her shortcomings, demanding affirmation for her illusory power. An adolescent is a young person who is developing into an adult. So how to help Natalie to develop an adult self-esteem when she fully identifies with her unrealistic fantasies?

Her grandiose fantasies are typical rather for a 7 year old girl than for a late adolescent. Should Natalie be told this despite her feeling of humiliation? Is the literal solution of the limited time of therapy enough for Natalie or should she be rather confronted with her aggression towards her Analyst and, in the transference, towards her parents? Isn't Natalie's Analyst just escaping from her patient's rage by giving her explanations instead of more difficult interpretations? Should she be even more flexible in her efforts to literally support Natalie or should she frustrate her more to encourage her to grow up?

The information obtained from the course of the therapy and the Analyst's counter-transferential feelings shows that 'Natalie' was interested very much in the feeling satisfaction and a retaliatory triumph which she gained from her enactment behaviour during the sessions. Thus, we can understand 'Natalie's' emotional difficulties as connected with a problem of perversion $[7,8]$. As it is usually in the case of this kind of problems, the Analyst experienced an inner pressure to identify and to act out, alternately, the role of a 'victim' and an 'oppressor', balancing between feelings of fear and guilt. Taking a role of the 'victim', she became 'too good mother' and in the role of the 'oppressor', she was sometimes 'too cruel shaman-guide.' As Linda Carter, she could say: "I had lost my grounding and feel powerless" [9].

The story about the house full of sweets, gives an impression that 'Natalie' grew up in the enchanted gingerbread house from the Grimm's' fairy tale "Hanzel and Gretel". As it was described by Bettelheim, the witch from the fairy tale is seen as so dangerous and hostile through the eyes of a child who is just preparing to leave a 'paradise' connected with an always available mother's breast [10]. In the case of 'Natalie', the situation was very complicated because, for her, the only known alternative for a mother who allowed her for everything was a father who humiliated and scared her.

The capacity to symbolise is achieved not only by playing but also by experiencing frustration caused by the situations that parents are not always accessible and not always fulfil their child's needs. In both our cases, there is a danger that therapists identifying with good parental figures and unconsciously competing with the patients' 'bad' parents, may slow down their patients' development by offering them too literal support.

It is also questionable whether a corrective experience of having a good parental figure in the person of a therapist is enough for our patients to go into adulthood and whether it will replace the exploration of their inner world. There is a question how much we can analyse our patients' relationships with both their real and intrapsychic parents in the situation when adolescents are still fully dependent on their parents emotionally and as well as financially and their parents pay for the therapy.

Because of their difficult history both patients have a problem with normal, adolescent, creative playing with thoughts and ideas. If they have ideas for their future, these ideas are rigid, fix and difficult to any shift. Also their anger to their parents it is not only adolescent rebellion against authority. Most adolescents need a confrontation with authority, it is normal phenomena. But their anger (or even rage) is much more powerful and connected with very difficult story with their parents from the past.

Both our patients' needs to rely on a real person (analyst) but at the same time analytical work should open a symbolic space where they found symbols which help them to rely on themselves.

\section{Conclusion}

The dilemma considered in this paper was: how much therapist is like a good 'foster parent' and how much like a 'shamanguide'. When we work with adolescents with deficits which derived from the time of childhood, being a good parent is important in order to enable the patient to build or re-build the reliable internal world. However, sometimes, a moment arises when frustration is necessary. This could be painful, like all the rituals of initiation are usually painful. These rituals allow young people to transit to the adult world. Often, our position as Analysts is balancing between playing a role of a 'good available mother' and a 'shaman-guide' who pushes an adolescent in a direction of the adult world and, through 
discovering individual symbols, helps them to struggle with life.

While worked on the text of our article, we came to the conclusion that the dilemma we were struggling with during the course of the therapy with our adolescent patients has not been developed sufficiently in the psychological literature. Therefore, with all the questions asked above, we would like to invite our colleagues and peers to share their experience and to continue the discussion regarding this crucial, in our opinion, topic.

\section{References}

1. Wiener J. The therapeutic relationship. Texas A\&M University Press. 2009.

2. Gordon R. Dying and creating: In a search for meaning. Symbols and symbols formation, Karnac Books Ltd., London. 1999.

3. Winnicott DW. The maturational processes and the facilitating environment. The Institute of Psycho-analysis Karnac Books. 1990;140-53.
4. Jung CG. Transcendent function. Collected Work 8, Bollingen Series. 1957.

5. Winnicott DW. Transitional objects and transitional phenomena: A study of the first not-me possession. Int $\mathbf{J}$ Psychoanal. 1953;34:89-97.

6. Klein M. The psychoanalysis of children. Hogarth Press, CW 2 London. 1945.

7. Ross F. Perversion. Karnac Books. 2003.

8. Ross F. Perversion: Jungian approach. Karnac Books. 2013.

9. Carter L. A Jungian contribution to a dynamic systems understanding of disorganised attachment. J Anal Psychol. 2011;56.

10. Bettelheim B. The uses of enchantment: The meaning and importance of fairy tales. Thames and Hudson. 1976. 